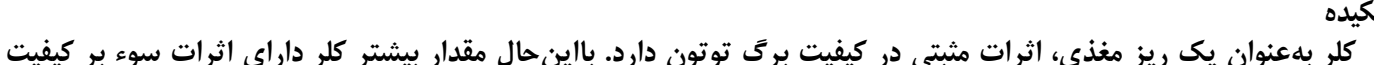

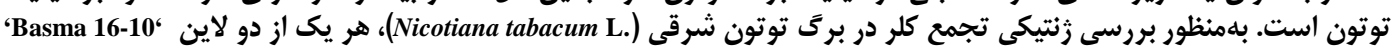

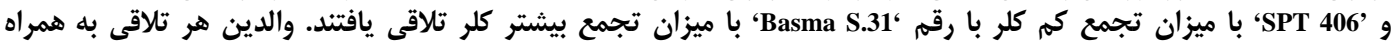

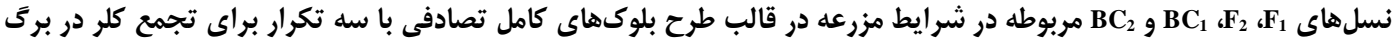

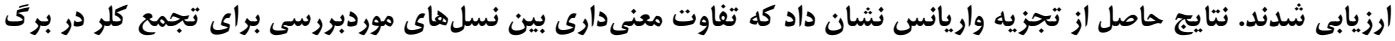

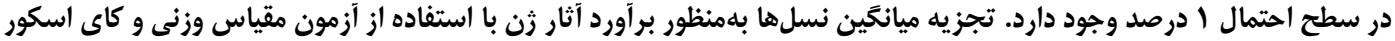

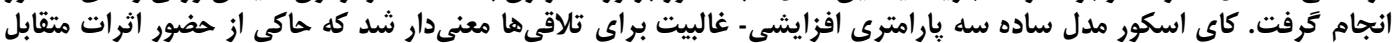

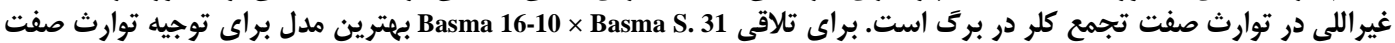

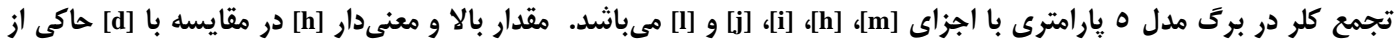

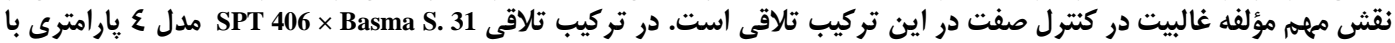

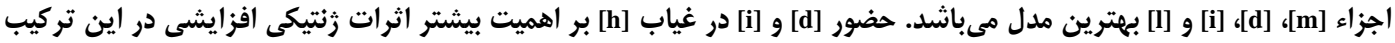

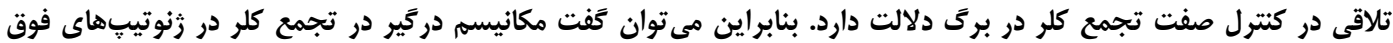

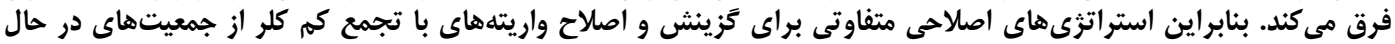
تفرق هريك از تلاقى هابـ لازم است.

وازههاى كليدى: تجزيه ميانكين نسلها، تجمع كلر، تنوع زنتيكى، توتون شرقى

سوزش و حفظ كيفيت برى توتون مى كود (سب). با اينحال

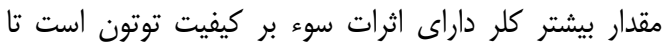

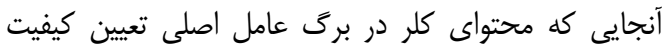

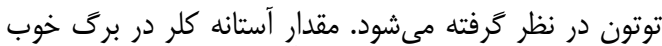

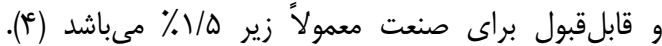

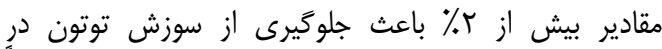

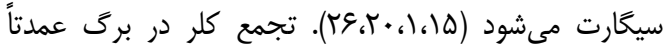

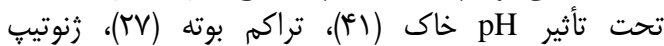

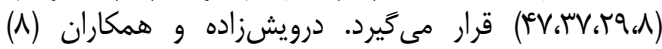

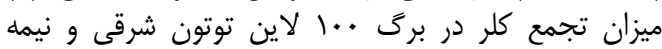

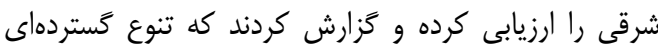

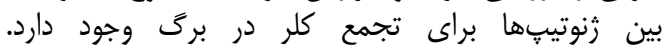

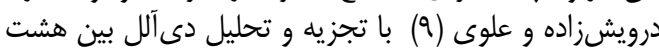

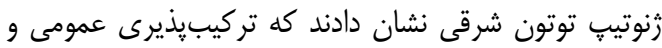

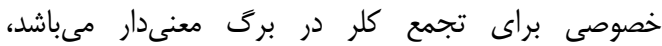

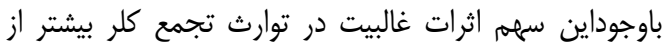

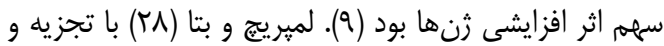

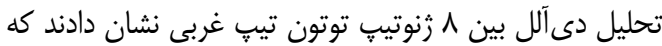

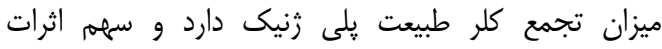

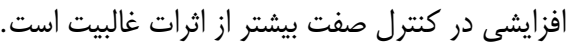

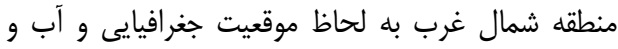

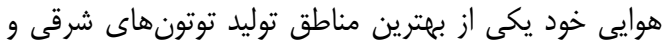

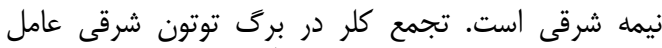

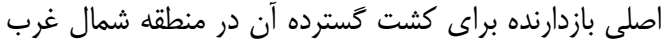

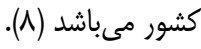

توتون (Nicotiana tabacum L.) يكى از محصولات با ارزش كشاورزى و صنعتى است و در اقتصاد كشورهاى

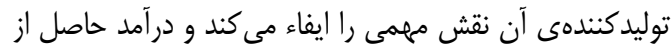

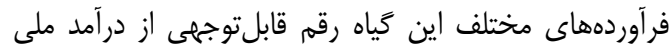

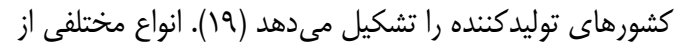

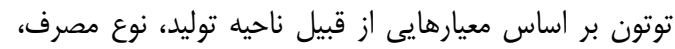

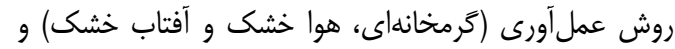

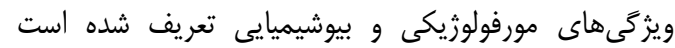

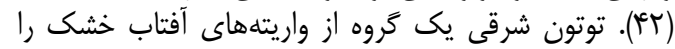

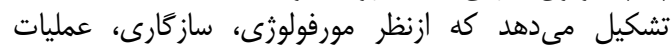

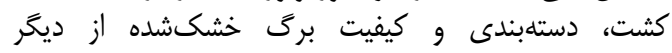

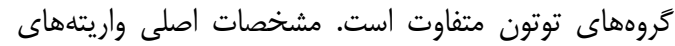

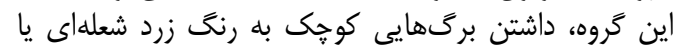

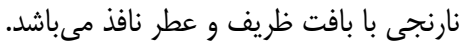

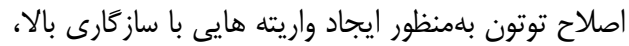

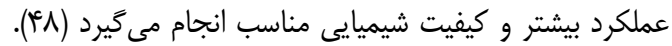

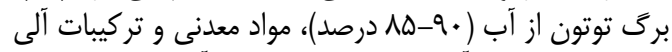

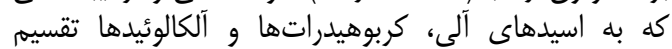

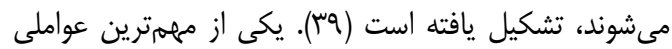

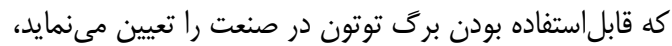

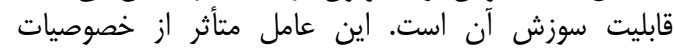

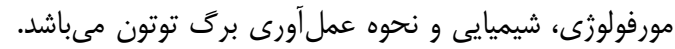

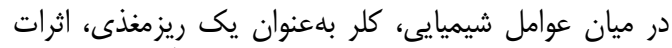

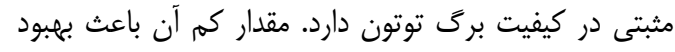

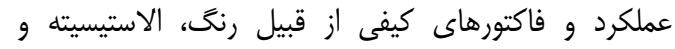




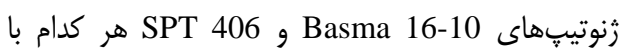
رنوتيب Basma S.31 در مركز تحقيقات توتون اروميه در

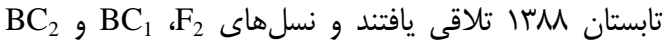

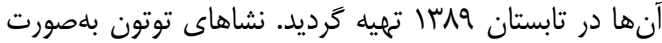

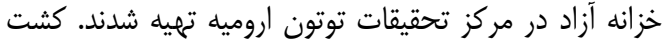

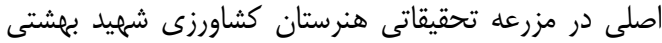

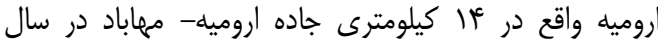

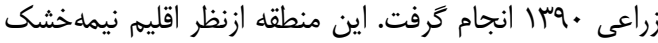

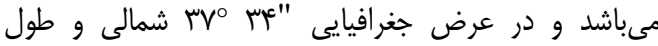

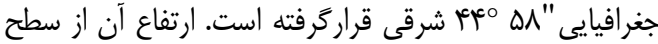
دريا

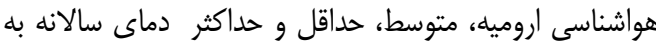

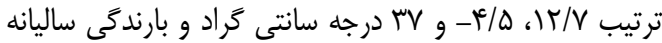

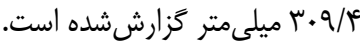

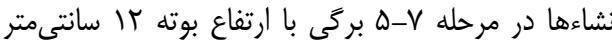

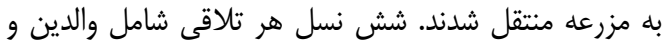

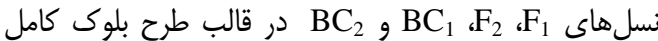
تصادفى با ب تكرار كشت شدند. در حقيقت هر خانواده (والدين

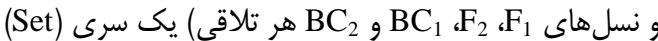

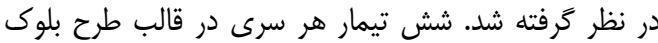

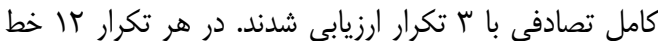

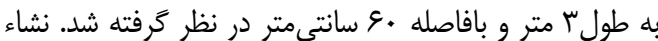

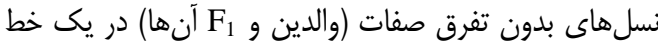
و نسلهاى در حال تفكيك (FC

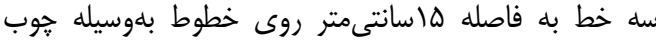

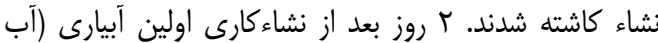

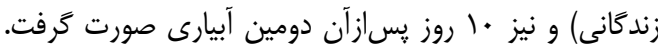

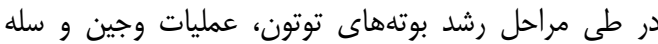

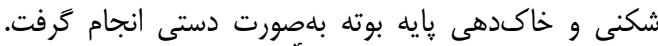

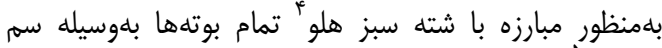

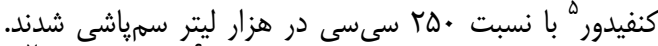

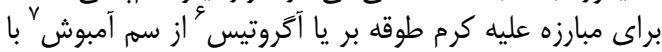

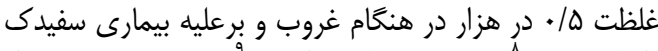

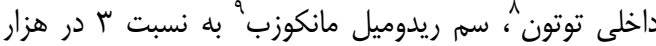

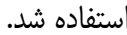

در طى مراحل رشد توتون برگهاى شند رسيده ازنظر صنعتى،

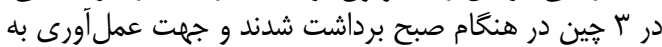

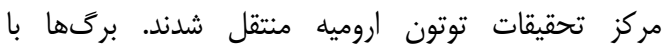

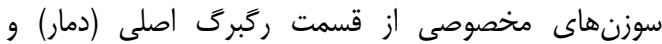

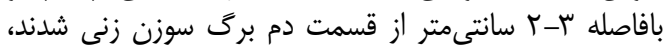

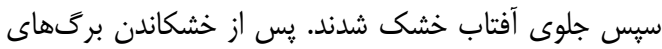

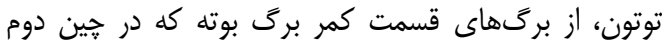

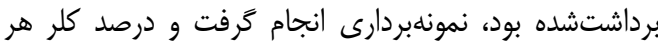

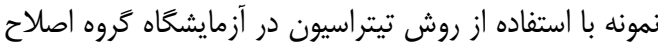

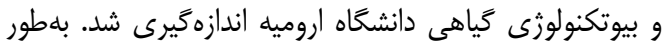

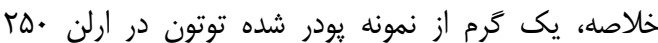

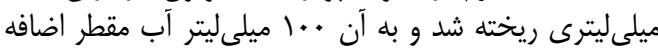

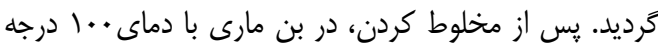

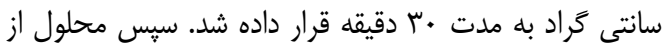

بهبود خاى و آب آبيارى بلمنظور كنترل سطح تجمع كلر

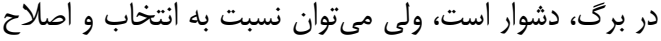

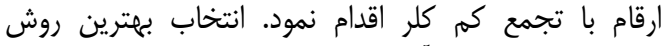

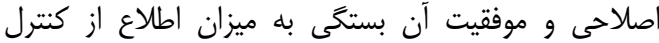

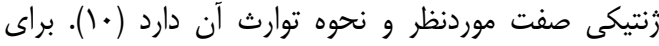

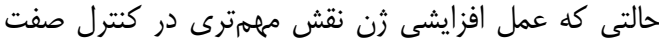

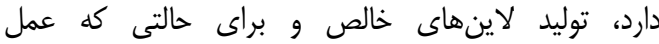

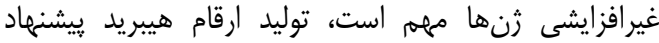

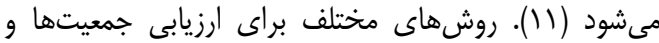

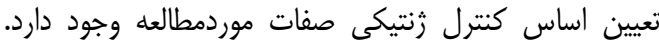

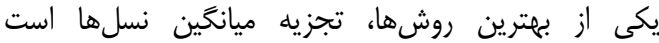

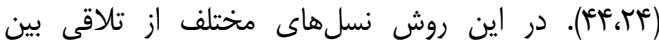

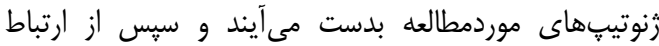

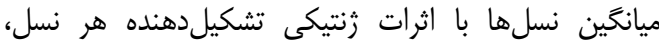

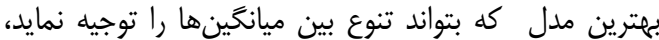

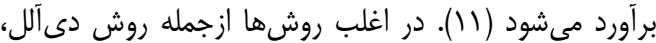

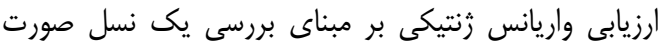

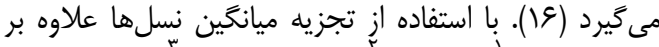

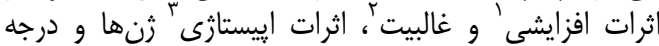

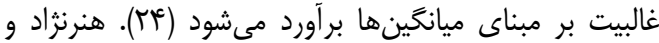

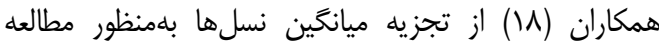

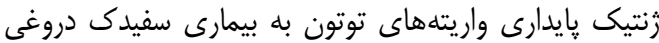

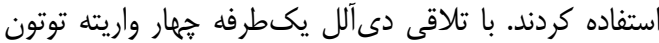

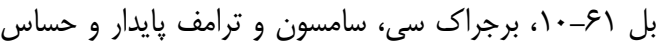
به بيمارى سفيدك دروغى و خودگشنى

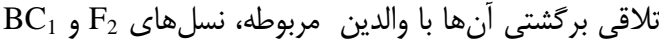

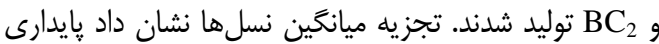

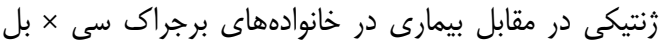

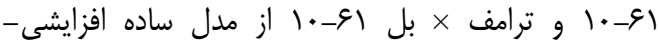

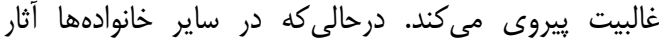

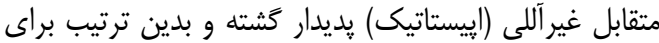

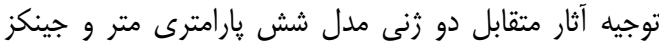

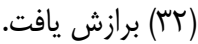

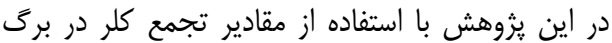

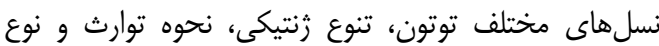

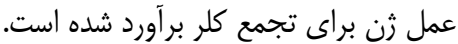

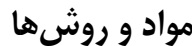

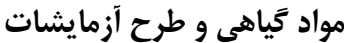

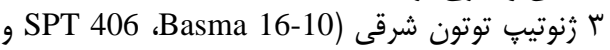
(Basma S. 31

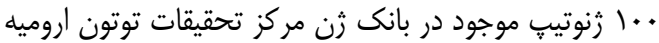

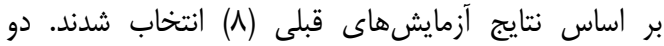

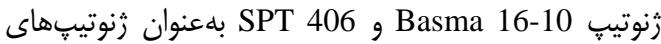
با تجمع كم كلر در برى و Basma S. 31 بهني ميزان تجمع بيشتر كلر در برى بر شناسايىشده بودند

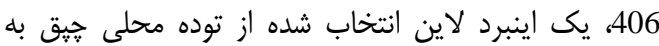

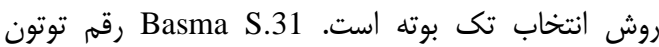
شرقى است كه در منطقه در سطح وسيع كشت مىشود.

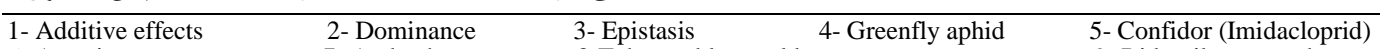

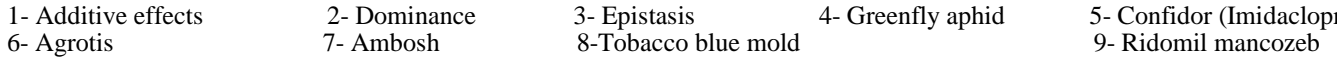




$$
\begin{aligned}
& \mathrm{h}_{b s}^{2}=\frac{\frac{1}{2}\left[\mathrm{~V}_{\mathrm{F} 2}-\left(\mathrm{V}_{\mathrm{P} 1} \times \mathrm{V}_{\mathrm{P} 1}\right)\right]}{\mathrm{V}_{\mathrm{F} 2}} \\
& \mathrm{~h}_{b s}^{2}=\frac{\frac{1}{3}\left[\mathrm{~V}_{\mathrm{F} 2}-\left(\mathrm{V}_{\mathrm{P} 1} \times \mathrm{V}_{\mathrm{P} 1} \times \mathrm{V}_{\mathrm{F} 1}\right)\right]}{\mathrm{V}_{\mathrm{F} 2}} \\
& \mathrm{~h}_{b s}^{2}=\frac{\frac{1}{3}\left[\mathrm{~V}_{\mathrm{F} 2}-\left(\mathrm{V}_{\mathrm{P} 1}+\mathrm{V}_{\mathrm{P} 1}+\mathrm{V}_{\mathrm{F} 1}\right)\right]}{\mathrm{V}_{\mathrm{F} 2}} \\
& \mathrm{~h}_{b s}^{2}=\frac{\frac{1}{2}\left[\mathrm{~V}_{\mathrm{F} 2}-\left(\mathrm{V}_{\mathrm{P} 1}+\mathrm{V}_{\mathrm{P} 1}\right)\right]}{\mathrm{V}_{\mathrm{F} 2}} \\
& \mathrm{~h}_{b s}^{2}=\frac{\frac{1}{4}\left[\mathrm{~V}_{\mathrm{F} 2}-\left(\mathrm{V}_{\mathrm{P} 1}+\mathrm{V}_{\mathrm{P} 1}+2 \mathrm{~V}_{\mathrm{F} 1}\right)\right]}{\mathrm{V}_{\mathrm{F} 2}}
\end{aligned}
$$

وراثتيذيرى خصوصى با استفاده از روش وارنر (\&ء) با استفاده از رابطه زير محاسبه شد.

$$
\mathrm{h}_{n s}^{2}=\frac{\left[2 \mathrm{~V}_{\mathrm{F} 2}-\left(\mathrm{V}_{\mathrm{BC} 1}+\mathrm{VBC} 2\right)\right]}{\mathrm{V}_{\mathrm{F} 2}}
$$

براى محاسبه حداقل تعداد زن هاى كنترل كننده صفت از روابط زير استفاده شد (V، •r).

$$
n=\frac{0.5\left(\mu_{P 2}-\mu_{P 1}\right)}{8\left(\delta_{F 2}^{2}-\delta_{F 1}^{2}\right)}
$$

$$
n=\frac{0.5\left(\mu_{P 2}-\mu_{P 1}\right)}{8\left[\delta_{F 2}^{2}-\left(0.5 \delta_{F 1}^{2}+0.25 \delta_{P 1}^{2}+0.25 \delta_{P 2}^{2}\right)\right]}
$$$$
n=\frac{0.5\left(\mu_{P 2}-\mu_{P 1}\right)}{8\left[\left(\delta_{B C 1}^{2}+\delta_{B C 2}^{2}\right)-\left(\delta_{F 1}^{2}+0.5 \delta_{P 1}^{2}+0.5 \delta_{P 2}^{2}\right)\right]}
$$

$$
n=\frac{0.5\left(\mu_{F 1}-\mu_{P 1}\right)}{4\left[\delta_{B C 1}^{2}-0.5\left(\delta_{F 1}^{2}+\delta_{P 1}^{2}\right)\right]}
$$$$
n=\frac{0.5\left(\mu_{P 2}-\mu_{F 1}\right)}{4\left[\delta_{B C 2}^{2}-0.5\left(\delta_{F 1}^{2}+\delta_{P 2}^{2}\right)\right]}
$$

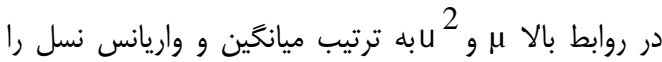

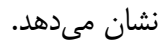

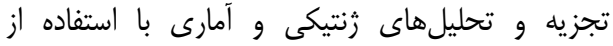

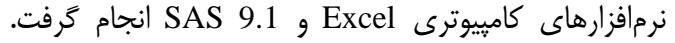
براى ترسيم نمودار توزيع فراوانى صفت در نسل F أز از نرمافزار SPSS 20
كاغذ صافى عبور داده شد. •ا ميلىليتر از نمونه فيلتر شده

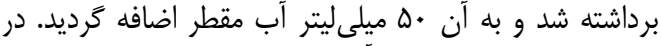

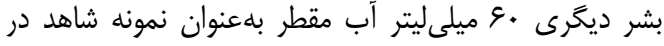

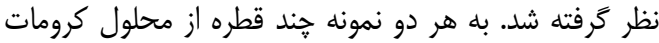

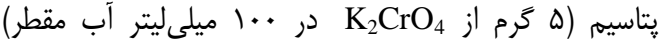

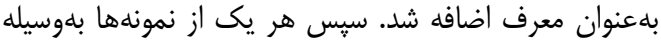

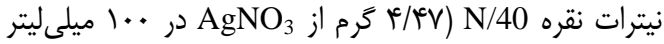

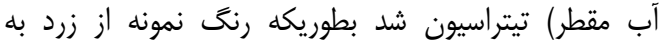
نارنجى سوخته تبديل شود و درصد كلر با استفاده از فرمول زير محاسبه شد.

\%Chlorine $=\frac{(A-B) \times f \times 35.5}{W \frac{(100-M)}{100}}$

:B مقدار AgNO استفادهشه براى هر نمونه توتون، مقدار AgNO

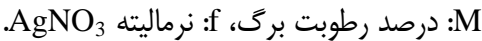

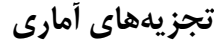

أزمون نرمال بودن توزيع اشتباهات آزمايشى مطابق روش

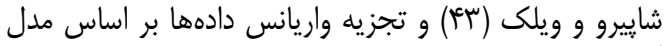

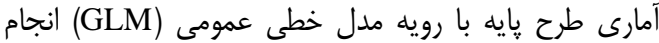
كرفت. تجزيه ميانكَين نسلها براب اساس مدل زير انجام كرفت.

$\mathrm{Y}=\mathrm{m}+\alpha \mathrm{d}+\beta \mathrm{h}+\alpha^{2} \mathrm{i}+2 \alpha \beta \mathrm{j}+\beta^{2} \mathrm{l}$

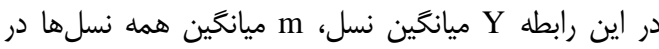

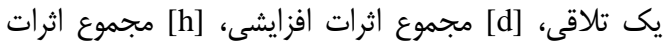

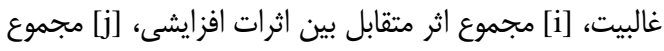

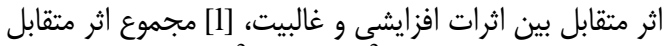

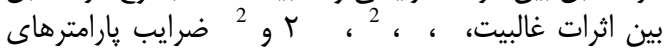

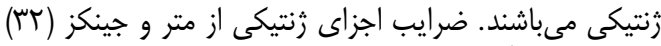

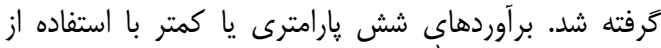

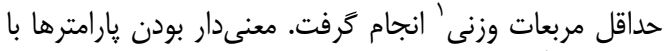

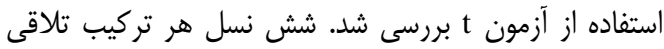

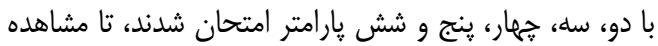

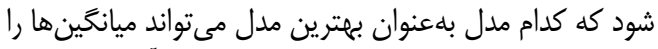

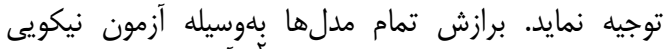

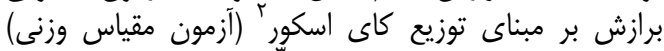

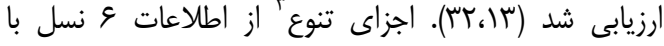

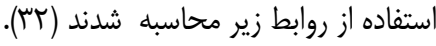

$\mathrm{E}_{\mathrm{w}}=\frac{1}{4}\left(V_{P 1}+V_{P 2}+2 V_{F 1}\right)$

$\mathrm{D}=4 \mathrm{~V}_{\mathrm{F} 2}-2\left(\mathrm{~V}_{\mathrm{BC} 1}+\mathrm{V}_{\mathrm{BC} 2}-\mathrm{E}_{\mathrm{w}}\right)$

$\mathrm{H}=4\left(\mathrm{~V}_{\mathrm{BC} 1}+\mathrm{V}_{\mathrm{BC}^{2}}-\mathrm{V}_{\mathrm{F}^{2}}-\mathrm{E}_{\mathrm{w}}\right)$

$\mathrm{F}=\mathrm{V}_{\mathrm{BC} 1}-\mathrm{V}_{\mathrm{BC} 2}$

در روابط بالا E جزء غير غيرزنتيكى تنوع، D جزء افزايشى

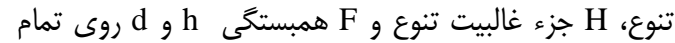

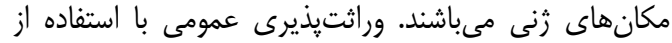


مى باشد. مقدار بالا و معنىدار اثر غالبيت [h] در مقايسه با اثر

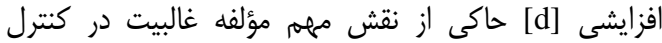

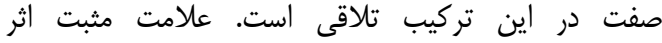

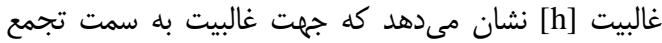

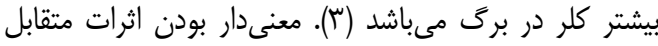

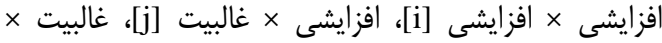

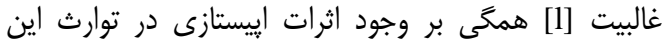

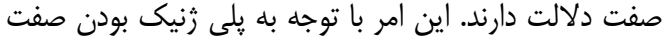

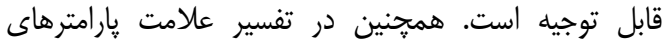

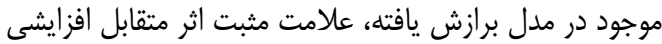

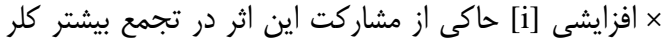

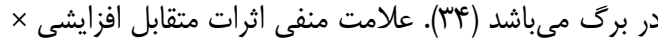

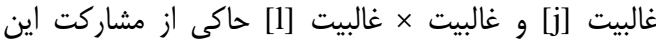

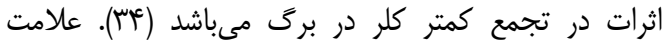

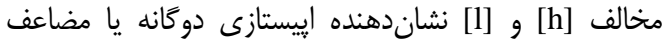

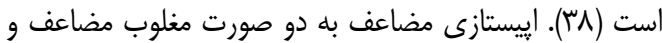

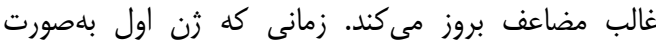

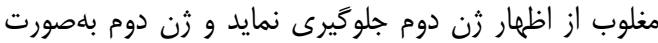

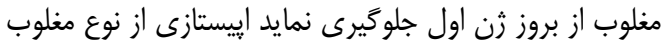

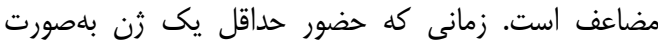

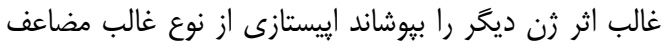

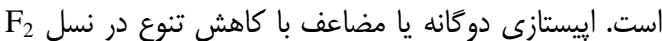

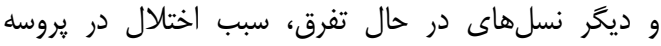

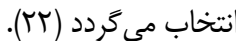

نتايج و بحث

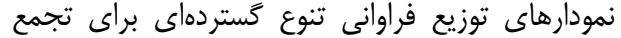

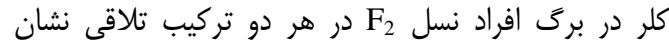

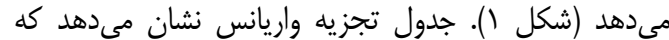

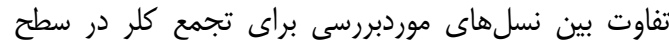

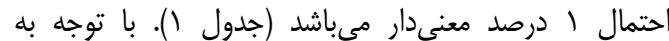

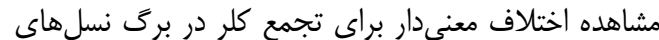

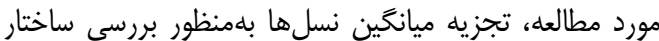

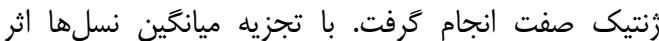

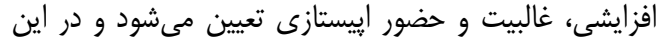

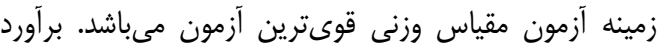

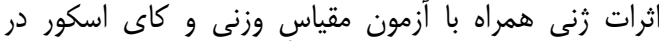

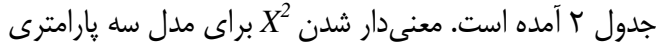

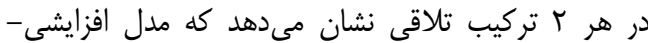

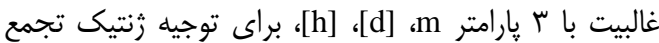

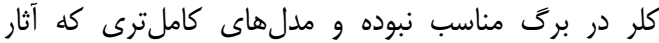

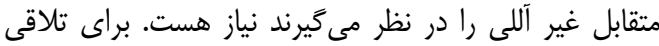
توارث مasma 16-10 × Basma S. 31

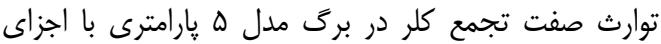

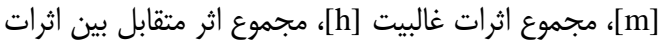

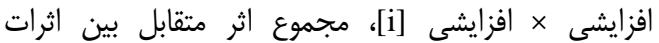

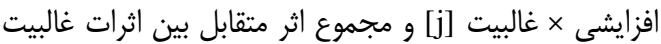

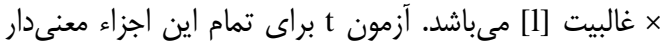

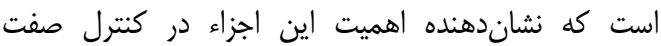

Bitsma $16-10 \times$ Basina $S .31$
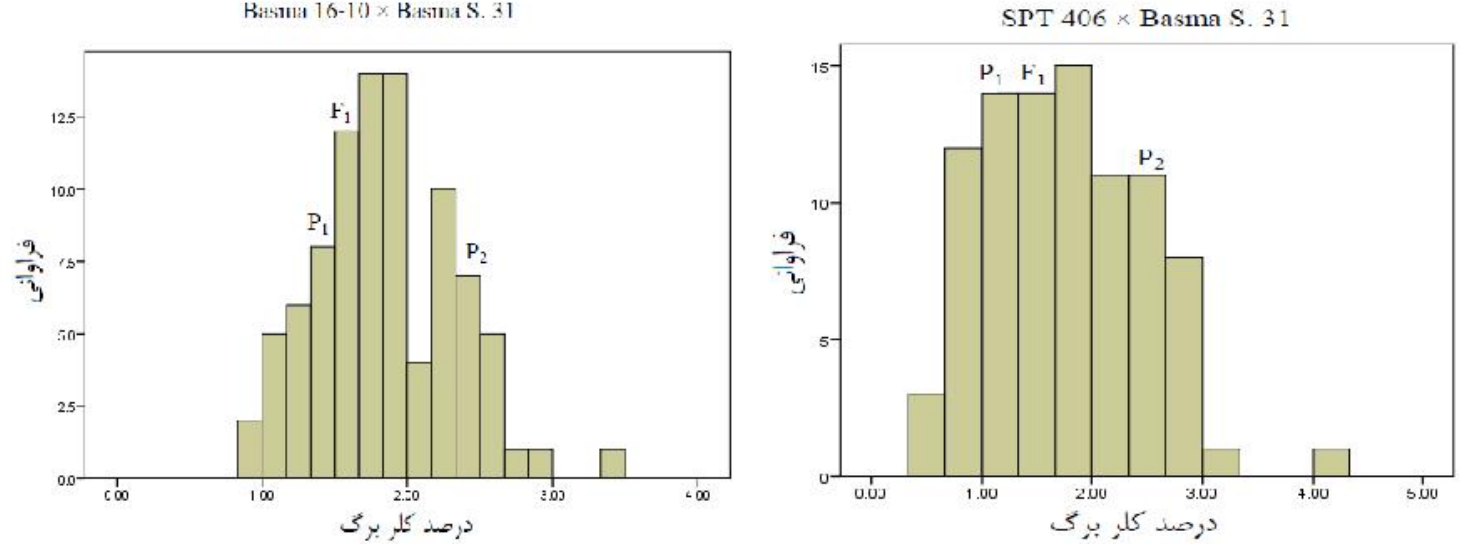

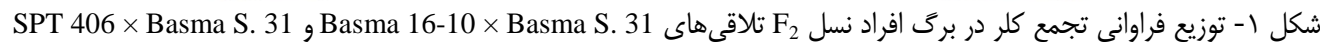
Figure 1. Frequency distribution of chlorine accumulation in the leaf of $F_{2}$ individuals of crosses Basma 16-10 $\times$ Basma S. 31 and SPT $406 \times$ Basma S. 31 . 


\begin{tabular}{|c|c|c|c|c|c|}
\hline \multirow{2}{*}{ مورد } & \multicolumn{4}{|c|}{ منابع تغييرات } & \multirow{2}{*}{$\% \mathrm{CV}$} \\
\hline & تكرار & سرى & سرى/ نسل & اشتباه آزمايشى & \\
\hline df & $r$ & 1 & 1. & Tr & \\
\hline MS & $\cdot / \cdot r$ &.$/ 1$ & $\cdot \mid+1$ & .1 .4 & $1 . / 11$ \\
\hline F & $\cdot / 98^{\mathrm{ns}}$ & $\cdot / r q^{\mathrm{ns}}$ & $|1 / F|^{* m}$ & & \\
\hline
\end{tabular}

جدول r- تخمين اجزاء زنتيكى ميانكين براى تجمع كلر در تلاقىهاى Basma S. 31 و Basma16-10 Basma S. 31 × BPT406 توتون

شرقى

Table 2. Estimate of genetic components of mean for chlorine accumulation in the crosses Basma 16-10 × Basma S.

\begin{tabular}{|c|c|c|c|c|c|c|c|}
\hline تلاقى & $\mathrm{m}$ & [d] & {$[\mathrm{h}]$} & [i] & [j] & [1] & $\mathrm{X}^{2}$ \\
\hline $\begin{array}{c}\text { Basma } 16-10 \times \text { Basma } \\
\text { S. } 31 \\
\text { SPT } 406 \times \text { Basma S. }\end{array}$ & $\begin{array}{l}\cdot / V \Lambda^{*} \pm \cdot / K^{\prime} \\
r / r^{* * *} \pm \cdot / \mu\end{array}$ & $-. / 9 \Delta^{* *}+\cdot / \cdot \Delta \mu$ & $\left.r / \mu f^{* * *} \pm \cdot / \Lambda\right)$ & $\begin{array}{c}1 / / r^{* *} \pm \cdot / \Psi r \\
-\cdot / \theta^{* *}{ }_{ \pm \cdot} / / \Lambda 1\end{array}$ & $-1 / \cdot F T^{* *} \pm \cdot / M A F$ & $\begin{array}{l}-1 / / \Lambda^{* *} \pm \cdot / \Delta \mid \\
-1 / r \Delta^{* *} \pm \cdot / r \Lambda\end{array}$ & $\begin{array}{l}\mathrm{ns} \\
\mathrm{ns}\end{array}$ \\
\hline
\end{tabular}

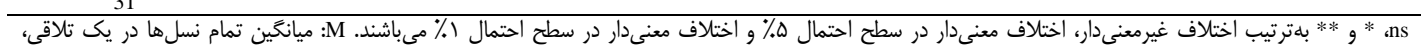

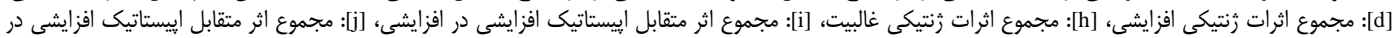
غالبيت، [1]: مجموع اثر متقابل إيبستاتيك غالبيت در غالبيتئ،

از نظر علامت و بزركى در مكانهاى مختلف با يكديخر

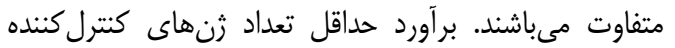

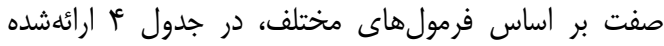

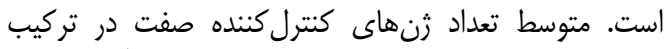

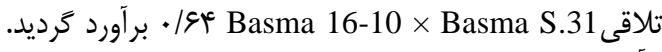
ازآنجايى كه روشهاى مختلف محاسبه حداقل تعداد زن، نياز

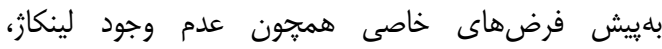

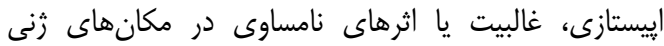

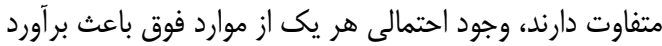
كمتر از حد واقع ثن هاى كنترل كننده صفت شده است (T) (I).

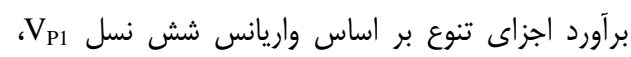

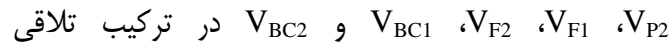
(H) نشان داد جزء غالبيت Basma16-10 Basma S.31

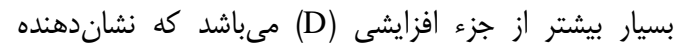

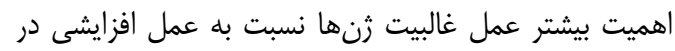

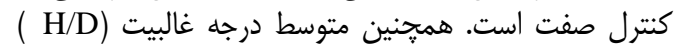

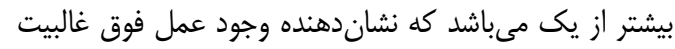

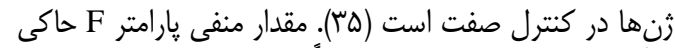

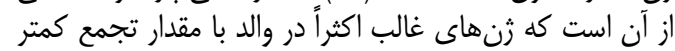

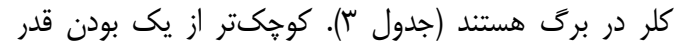

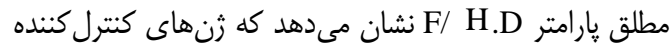

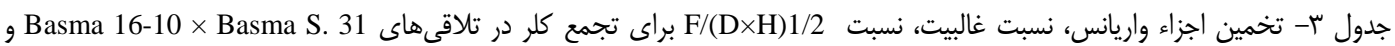
SPT 406 × Basma S.31

Table 3. Estimates of the variation components, dominance ratio, $\mathrm{F} /(\mathrm{D} \times \mathrm{H}) 1 / 2$ ratio for chlorine accumulation in the crosses Basma 16-10 $\times$ Basma S. 31 and SPT $406 \times$ Basma S. 31 of oriental tobacco

\begin{tabular}{|c|c|c|c|c|c|c|}
\hline تلاقى & $\mathrm{E}_{\mathrm{W}}$ & $\mathrm{F}$ & $\mathrm{H}$ & $\mathrm{D}$ & $\sqrt{\mathrm{H} / \mathrm{D}}$ & $\mathrm{F} / \mathrm{NH} . \mathrm{D}$ \\
\hline Basma 16-10 × Basma S. 31 & $\cdot 1 \cdot 1$ &.$- / .4$ & $\cdot / 4 \mid$ & $\cdot 1 \cdot 0$ & $r / A \Gamma$ & $-\cdot / 1 \pi$ \\
\hline SPT $406 \times$ Basma S. 31 & $\cdot / r \Delta$ & . & .109 &.$/ T V$ & $1 / \pi 9$ & س سو/. \\
\hline
\end{tabular}

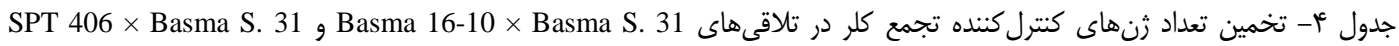

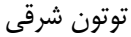

Table 4. Estimates of number of genes controlling chlorine accumulation trait in the crosses Basma 16-10 × Basma S. 31 and SPT $406 \times$ Basma S. 31 of oriental tobacco

\begin{tabular}{|c|c|c|c|c|c|c|}
\hline \multirow[b]{2}{*}{ 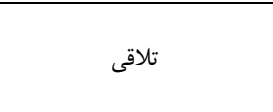 } & \multicolumn{5}{|c|}{ روش } & \multirow[b]{2}{*}{ ميانگين } \\
\hline & $\stackrel{\text { I }}{\text { Cockerham }(V)}$ & $\begin{array}{c}\text { II } \\
\text { Cockerham (V) }\end{array}$ & $\begin{array}{c}\text { III } \\
\text { Lande } \\
(\mu \cdot)\end{array}$ & $\begin{array}{c}\text { IV } \\
\text { Lande } \\
(\mu \cdot)\end{array}$ & $\begin{array}{c}\mathrm{V} \\
\text { Lande } \\
(\mu \cdot)\end{array}$ & \\
\hline Basma 16-10 × Basma S. 31 & $\cdot|\wedge|$ &.$/ 9$. &.$/ \Delta$ &.$/ 4 V$ &.$/ \Delta F$ &.$/ 94$ \\
\hline SPT $406 \times$ Basma S. 31 & $1 / T F$ & $1 / .9$ & $\cdot / V f$ & $\cdot 1 \cdot V$ & D/\& & $1 / V \Delta$ \\
\hline
\end{tabular}

زنتيكى) براى صفت در مقايسه با بخش غير افزايشى بسيار

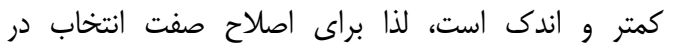
نسلهاى بيشرفته اين تركيب تلاقى نتايج خوبى بله همراه خواهد داشت.

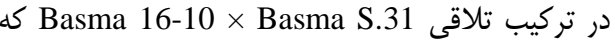

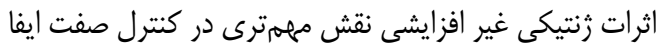

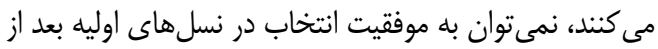

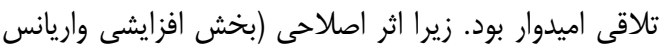




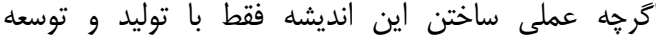

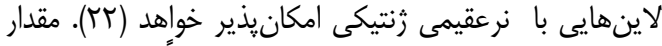

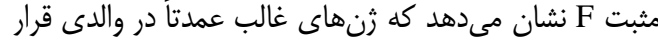

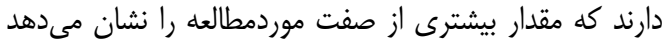
F/fl

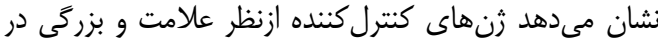

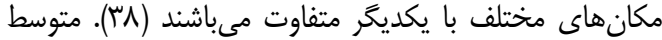

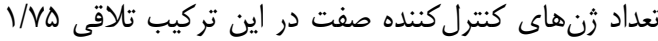

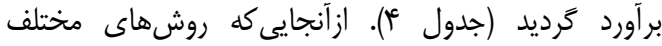

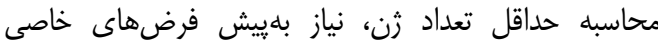

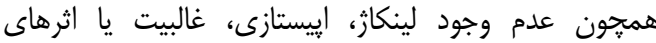

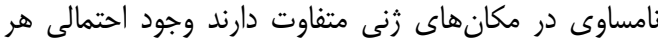

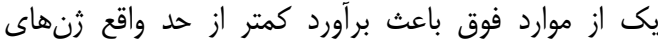
كنترل كننده صفت شده است (I) برد

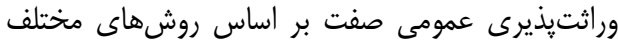
در تركيب تلاقى اول (Basma 16-10 × Basma S.31)

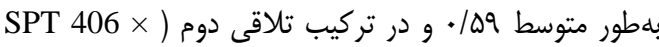
(Basma S. 31

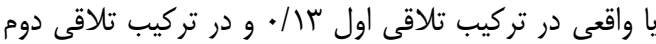

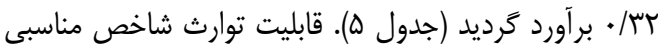

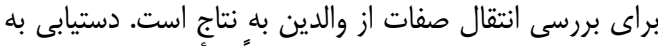

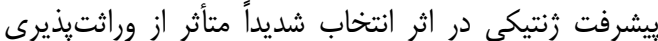

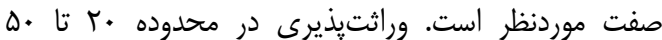

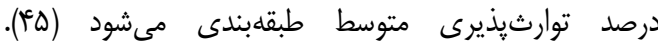

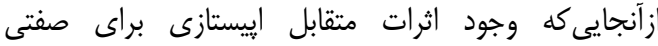

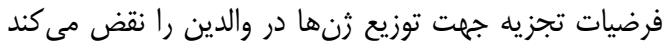

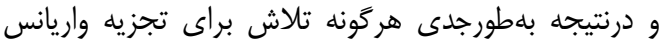

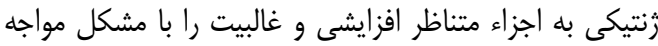

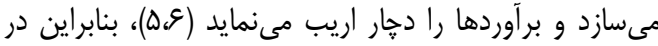

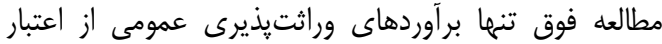

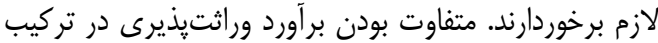

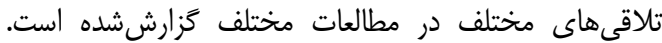

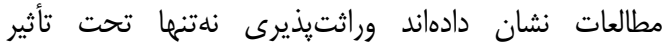

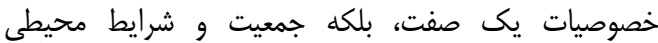

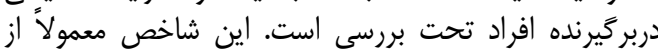
جمعيتى به جمعيت ديخر متفاوت است است است است

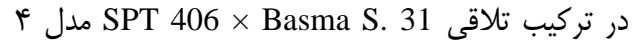

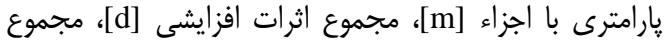

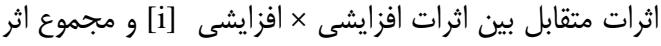

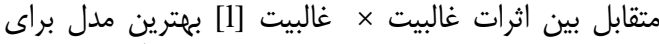

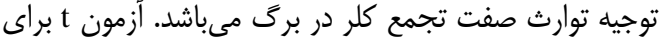

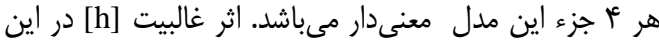

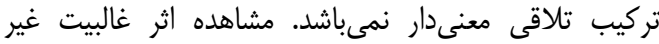

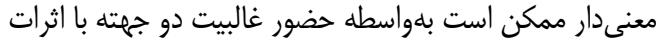

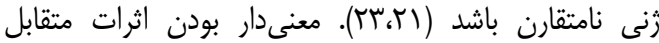

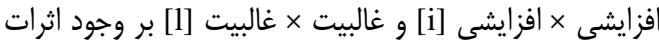

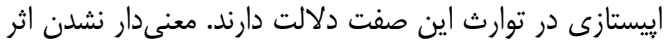

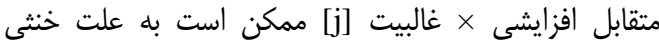

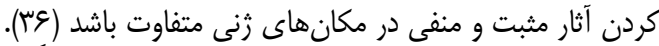

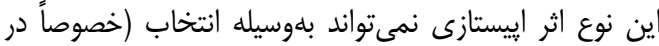

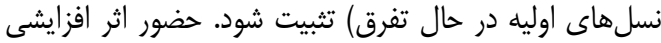

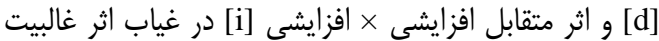

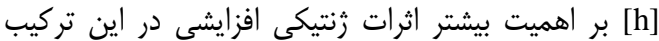

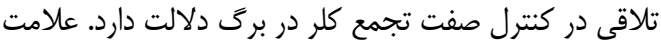

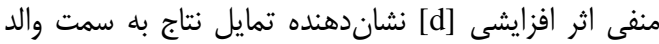

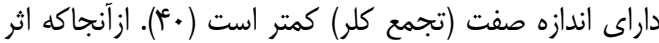

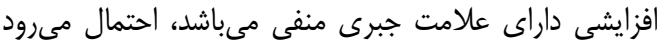

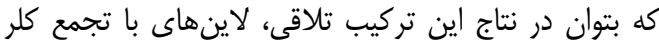

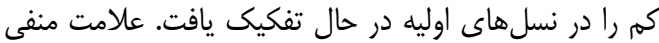

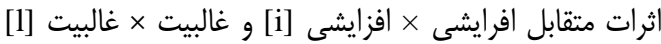
حاكى از مشاركت اين اثرات در تجمع افرايشى كم كلر در برى مىباشد.

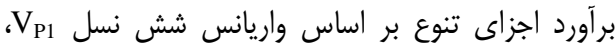

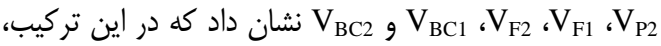

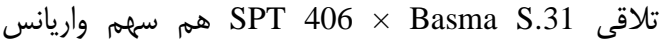

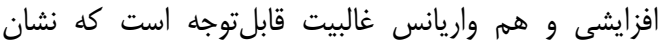

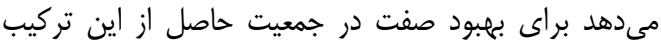

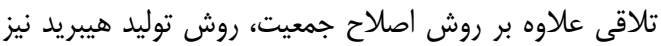

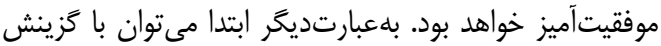

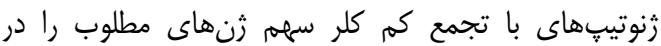

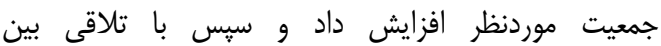
زنوتيبهاى انتخابى هيبريدهاى با تجمع كمتر كلر توليد نمود.

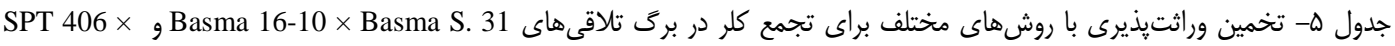
Basma S. 31

Table 5. Estimates of the heritability by different methods for chlorine accumulation trait in leaf of the crosses Basma 16-10 × Basma S. 31 and SPT406 $\times$ Basma S. 31 of oriental tobacco

\begin{tabular}{|c|c|c|c|c|c|c|c|}
\hline \multirow[b]{2}{*}{ 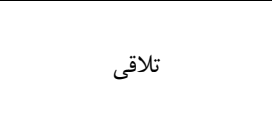 } & \multirow{2}{*}{$\begin{array}{c}h_{n s}^{2} \\
\text { Warnner } \\
(\leftarrow \varepsilon)\end{array}$} & \multicolumn{5}{|c|}{$h_{b s}^{2}$} & \multirow[b]{2}{*}{ ميانگين } \\
\hline & & $\begin{array}{c}\text { I } \\
\text { Mahmud \& Kramer (w)) }\end{array}$ & $\begin{array}{c}\text { II } \\
\text { Warnner } \\
(\leftarrow \varepsilon)\end{array}$ & $\begin{array}{c}\text { III } \\
\text { Allard } \\
(\tau)\end{array}$ & $\begin{array}{c}\text { IV } \\
\text { Allard } \\
(\Gamma)\end{array}$ & $\begin{array}{c}\mathrm{V} \\
\text { Mather \& Jinks } \\
(\mathrm{YY})\end{array}$ & \\
\hline Basma $16-10 \times$ Basma S. 31 &.$/ 1 \pi$ & $\cdot / \Delta \varphi$ & - &.$/ 9$. & $\cdot / \Delta g$ & 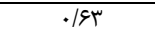 &.$/ 09$ \\
\hline SPT $406 \times$ Basma S. 31 & 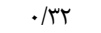 & .199 & - & $\cdot 19$. & .199 & $\cdot / \Delta V$ & . /at \\
\hline
\end{tabular}




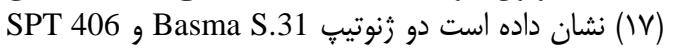

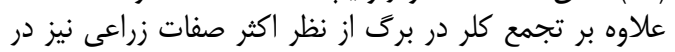

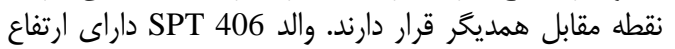

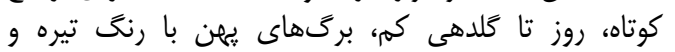

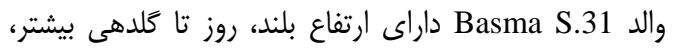

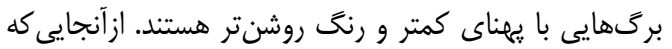

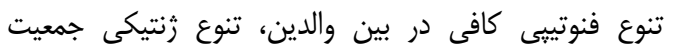

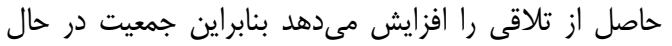

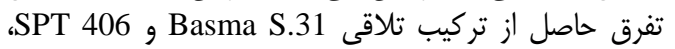

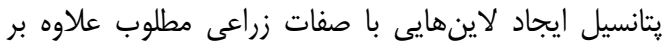

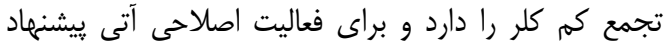
مى شود.
تحقيقات توتون اروميه انتخاب شدهاند. نتايج تحقيقات قبلى دوانى

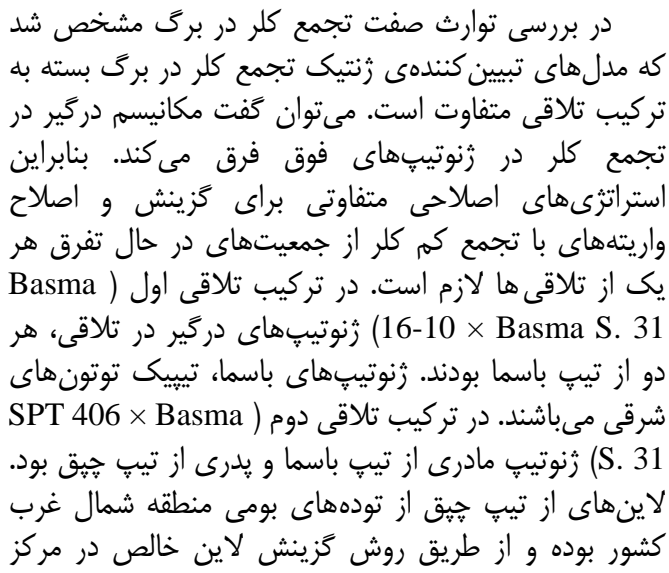

منابع

1. Akehurst, B.C. 1981. Tobacco $2^{\text {nd }}$ edition Tropical Agricultural Series. New York: Longman Inc. 164 2. Allard, R.W. 1960. Principles of Plant Breeding, $2^{\text {nd }}$ edition John Wily and Sons, Inc. New York. 254 pp.

3. Babaei, H.R., H. Zeinali Khanghah and A. Taleei. 2012. Genetic analysis of agronomic traits and seed shattering resistance in soybean (Glycine max). Seed and Plant Improvement Journal, 28(4): 593-609 (In Persian).

4. Chari, M.S. 1995. Role of research in the improvement of productivity and quality of Indian flue-cured Virginia tobacco. Central Tobacco Research Institute, Rajahmundry, India. 26-27 pp.

5. Choukan, R., H. Abtahi and E. Majidi Hervan. 2007. Genetic analysis of different traits in maize using diallel cross analysis. Iranian Journal of Crop Sciences, 8(4): 343-356 (In Persian).

6. Choukan, R. 2008. Methods of Genetical Analysis of Quantitative Traits in Plant Breeding. 1st edition. Agricultural Research, Education and Extension Organization, Tehran, Iran. 270 pp (In Persian).

7. Cokerham, C.C. 1988. Modification in estimating the number of genes for a quantitative character. Genetics, 114: 659-664.

8. Darvishzadeh, R., R. Alavi and A. Sarrafi. 2011. Genetic variability for chlorine concentration in oriental tobacco genotypes. Archive of Agronomy and Soil Science, 57(2): 167-177.

9. Darvishzadeh, R. and R. Alavi. 2011. Genetic analysis of chlorine concentration in oriental tobacco. Journal of Plant Nutrition, 34: 1070-1078.

10. Dixit, G.P. 1998. Gene action for yield and its components in grass pea. Indian Journal of Genetics, 58: 91-95.

11. Farshadfar, E. 2000. Application of Quantitative Genetics in Plant Breeding. Tagh-E- Bostan Press, Kermanshah, Iran. 726 pp (In Persian).

12. Ghannadha, M.R. 1998. Gene action for latent period of stripe rust in five cultivars of wheat. Iranian Journal of Crop Sciences, 1: 53-71 (In Persian).

13. Ghannadha, M.R. 2000. Gene functiona for resistance to yellow rust in wheat. Iranian Journal of Crop Sciences, 3: 397-407 (In Persian).

14. Golparvar, A.R., I. Majidi-Haravan, F. Darvish, A.M. Rezaie and A. Ghasemi Pirbalouti. 2004. Genetic assessment of some morpho-physiological traits in bread wheat under drought stress conditions. Pajouhesh \& Sazandegi, 62: 90-95 (In Persian).

15. Guardiola, J.M., O. Perez and L. Diaz. 1987. Effect of chlorine and potassium on combustibity from fine plantations. Tobacco, 10: 29-43.

16. Hallauer, A.R. and J.B. Miranda. 1982. Quantitative Genetic in Maize Breeding. The Iowa State University Press. Ames, Iowa. 680 pp.

17. Hatami Maleki, H., Gh. Karimzadeh, R. Darvishzadeh and R. Alavi. 2012. Genetic variation of oriental tobaccos using multivariate analysis. Iranian Journal of Field Crops Research, 10(1): 100-106 (In Persian).

18. Honarnejad, R., M. Shoai Deylami and M. Mesbah. 2001. Genetics of resistance to blue mould (Peronospora tabacina Adam) of tobacco. Journal of Science and Technology of Agriculture and Natural Resources, 5(2): 65-74 (In Persian).

19. Hosseinzadeh Fashalami, N., Z. Shahadati Moghaddam, Gh. Kiani, M.R. Salavati, P. Zamani, A.R. Mahdavi and R. Alinejad (2015). Investigation of genetic diversity among different oriental tobacco (Nicotiana tabacum L.) varieties using multivariate methods. Journal of Crop Breeding, 7(15): 126134 (In Persian).

20. Juan, R. and N. del Castillo. 1986. Irrigation water management and chemical and physical characteristics of covered dark tobacco. Riego y Drenaje, 9: 71-83.

21. Kamalizadeh, M., A.H. Hosseinzadeh and H. Zeinali Khaneghah. 2013. Evaluation of inheritance for some quantitative traits in bread wheat using generation means analysis under water deficit condition. Iranian Journal of Crop Sciences, 44(2): 317-326 (In Persian). 
$1 \%$. بررسى زنتيكى تجمع كلر در برگ توتون شرقى

22. Karami, E., S.H. Sabagh Pur, M.R. Naghavi and M. Taeeb. 2011. Genetic analysis of earliness in chickpea (Cicer arietinum L.) using generation mean analysis. Iranian Journal of Pulses Research, 2(2): 63-68 (In Persian).

23. Karami, E. 2011. Genetic analysis of drought tolerance in chickpea (Cicer arietinum L.) using generation mean analysis. Journal of Crop Sciences, 42(1): 165-182 (In Persian).

24. Kearsey, M. and H.S. Pooni. 1996. The Genetical Analysis of Quantitative Traits. London: Chapman and Hall, $381 \mathrm{pp}$.

25. Kiani, Sh., N. Babaeian Jelodar, Gh. Ranjbar, S. K. Kazemitabar and M. Nowrozi. 2015.The genetical evaluation of quantitative traits in rice (Oryza sativa L.) by generation mean analysis. Journal of Crop Breeding, 7(15): 105-114 (In Persian).

26. King, M.J. 1990. Tobacco. In: Stewart, B.A. and D.R. Nielsen, editors. Irrigation of Agricultural Crops. Agronomy Series, vol. 30. Madison (WI): American Society of Agronomy Inc. pp: 811-833.

27. Lamprecht, M.P., F.J. Shaw, C.J.H. Pretorius, A.H. Botha, M.C. Beer and J.G. Nel. 1979. The effect of spacing and topping on some quality components of low-profile flue-cured tobacco. Agroplanta, 11: $35-39$.

28. Lamprecht, M.P. and A.H. Botha. 1975. Genetic basis of chlorine concentration in flue-cured tobacco. Agroplanta, 7: 25-30.

29. Lamprecht, M.P. and C.J. Steenkamp. 1972. Difference in chlorine content of flue-cured tobacco cultivars. Agroplanta, 4: 69-72.

30. Lande, R. 1981. The minimum number of genes contributing to quantitative variation between and within populations Genetics, 95: 541-553.

31. Mahmud, I. and H. Krammer. 1951. Segregation for yield, height and maturity following a soybean cross. Agronomy Journal, 43: 605-609.

32. Mather, K. and T.L. Jinks. 1982. Biometrical Genetics. $3^{\text {nd }}$ edition. Chapman \& Hall. London, 396 pp.

33. McEvoy, E.T. 1957. The growth and mineral content of flue-cured tobacco as influenced by reaction of nutrient solutions with ionic forms of nitrogen Canadian Journal of Soil Science, 37: 79-83.

34. Mohammadi, M., S.S. Ramzanpour, S. Navabpour, H. Soltanloo, M. Kalate Arabi and Sh. Kia. 2012. Study on inheritance of resistance to Septoria tritici Blotch of wheat by generation means analysis. Journal of Plant Production, 19(4): 1-18 (In Persian).

35. Mostafavi, K.H., A.H. Hosseinzadeh, H. Zeinali Khaneghah and M. Khalou Bagheri. 2005a. Genetics of resistance to Sunn pest (Eurygaster integriceps Put.) in bread wheat. Iranian Journal of Agricultural Sciences, 36(2): 341-351 (In Persian).

36. Mostafavi K.H., A.H. Hosseinzadeh and H. Zeinali Khaneghah. 2005b. Genetic analysis of yield and correlated traits in bread wheat (Triticum aestivum). Iranian Journal of Agricultural Sciences, 36(1): 187-197 (In Persian).

37. Myhre, D.L., O.J. Attoe and W.B. Ogden. 1956. Chlorine and other constituents in relation to tobacco leaf-burn. Soil Science, 20: 547-551.

38. Nakhjavan, S., M.R. Bihamta, F. Darvish, B. Sorkhi and M. Zahravi. 2012. Heritability of agronomic traits in the progenies of a cross between two drought tolerant and susceptible barley genotypes in terminal drought stress conditions. Iranian Journal of Crop Sciences, 14(2): 136-154 (In Persian).

39. Provost, A. 1959. Technique du Tabac. Lausanne: Holiographic S.A. 331 pp.

40. Rabiei, B. and A. Ghorbanipour. 2011. Assessment of gene action and heritability of important agronomic traits in rice (Oryza sativa L.) using generation mean analysis. Iranian Journal of Crop Sciences, 13(2): 408-423 (In Persian).

41. Reisenauer, H.M. and W.E. Colwell. 1950. Some factor affecting the absorption of chlorine by tobacco. Soil Science, 15: 222-229.

42. Ren, N. and M.P. Timko. 2001. ALFP analysis of genetic polymorphism and evolutionary relationships among cultivated and wild Nicotiana species. Genome, 44: 559-571.

43. Shapiro, S.S. and M.B. Wilk. 1965. An analysis of variance test for normality. Biometrika, 52: 591599.

44. Singh, R.P. and S. Singh. 1992. Estimation of genetic parameters through generation mean analysis in bread wheat. Indian Journal of Genetics, 52: 369-375.

45. Stansfield, W.D. 1991. Theory and Problems in Genetics. McGraw-Hill. 452 pp.

46. Warnner, J.N. 1952. A method for estimating heritability. Agronomy Journal, 44: 427-430.

47. Williamson, R.E. and J.F. Chaplin. 1981. Levels of chemical constituents in cured leaves of four burley tobacco cultivars according to stalk position. Tobacco Science, 25: 75-78.

48. Yang, B.C., B.G. Xiao, X.J. Chen and C.H. Shi. 2007. Assessing the genetic diversiy of tobacco germplasm using inter simple sequence repeat and inter-retrotransposon amplification polymorphism markers. Annals of Applied Biology, 50: 393-40. 


\title{
Study on Genetic of Chlorine Accumulation in Leaves of Oriental Tobacco (Nicotiana tabacum L.)
}

\author{
Reza Darvishzadeh ${ }^{1}$, Mir Javad Mousavi Andazghi ${ }^{2}$ and Amir Fayyaz Moghaddam ${ }^{3}$ \\ 1- Professor, Urmia University, (Corresponding author: r.darvishzadeh@urmia.ac.ir) \\ 2 and 3- Gratuated M.Sc. Student and Associate Professor, Urmia University \\ Received: December 4, 2015 \\ Accepted: June 1, 2016
}

\begin{abstract}
Chlorine as a micronutrient has a positive effect on the quality of tobacco leaves. However, the more chlorine has adverse effects on tobacco quality. To study the genetic accumulation of chlorine in leaves of oriental tobacco, each of the two lines 'Basma 16-10' and 'SPT 406' with the low accumulation of chlorine were crossed separately with Basma S. 31 with the high accumulation of chlorine. Parents of each cross together with $\mathrm{F}_{1}, \mathrm{~F}_{2}, \mathrm{BC}_{1}$ and $\mathrm{BC}_{2}$ generations were evaluated for chlorine accumulation in a randomized complete block design with 3 replications under filed condition. Analysis of variance showed a significant difference between generations for accumulation of chlorine in leaves. Therefore, generation mean analysis was performed to estimate gene actions using Chi-square and scaling tests. The Chi-square of simple three-parametric model (additive- dominance model) was significant for studied crosses that indicate the presence of non-allelic interaction in the inheritance of property of accumulation of chlorine in leaves. In 'Basma S. $31 \times$ Basma 16-10' cross, the best model to explain the inheritance of chlorine accumulation is 5-parameters model with [m], [h], [i], [j] and [l] components. High and significant value of [h] compared to [d] show the importance of dominance effect in controlling trait. In 'SPT406 $\times$ Basma S. 31' cross, 4-parameters model with [m], [d], [i], and [1] components is the best model. The presence of [d] and [i] in the absence of [h] suggest the importance of additive genetic effects in control of chlorine. So we can say that the mechanisms involved in the accumulation of chlorine are different in studied genotypes. So, different breeding strategies are necessary to selection and modification of genotypes with low chlorine accumulation in segrigation populations.
\end{abstract}

Keywords: Chlorine accumulation, Generation mean analysis, Genetic diversity, Oriental tobacco 